\title{
The Orbit Design and Control Strategy of The Soft Landing of Chang 'e 3 Yan $\mathrm{YaO}^{1, \mathrm{a}}$, Yingbing $\mathrm{Fan}^{1}$ and Xiaocheng $\mathrm{Gao}^{2}$ \\ ${ }^{1}$ Department of Mathematics, Heihe University, Heilongjiang 164300, China \\ ${ }^{2}$ Office of Academic Affairs, Heihe University, Heilongjiang 164300, China \\ ahyaoyan@126.com
}

Keywords: the soft landing, the Hermite interpolation method, the newton interpolation

\begin{abstract}
In this paper, we solve the problem of the orbit design and control strategy of the soft landing of Chang'e 3,Use the Hermite interpolation method and the Parabolic interpolation method to analyze each stage of the process.By using the interpolation method in numerical analysis, which based on the theory of kinematics and mechanics knowledge, with the help of the Realization of matlab sortware to solve the problem.
\end{abstract}

\section{Introduction}

The development of the lunar exploration program for Chinese people,Which marks the progress of science and technology of China. And the successful of the landing for Chang 'e 3, means more realization for the first time in our country.And this will be the first time for our country's spacecraft in the extraterrestrial objects to a soft landing and patrol survey, It has an important significance to promote the innovation and development of China's space technology.

Chang'e 3 was successful launched at 1:30 on December 2, 2013 .And arrived in lunar orbit On December 6. Chang 'e 3 in the case of high-speed flight, To ensure a accurately soft landing on the accurate reservation area on the moon.

The key question is the landing trajectory and the strategy of the control design.The soft landing process is divided into six stages,It required to meet the state at the key points of each stage . to minimize the fuel consumption of the process of the soft landing and to make sure the moon landing orbit and the optimal control strategy at the 6 stages .

\section{The optimal control strategy of Chang 'e 3 soft landing trajectory}

The moon landing trajectory of Chang'e 3. In order to achieve a soft landing on the moon,It must make the impact of the lander and the relative velocity of the moon to be the landing buffer which can be absorbed by the buffering devices ,To ensure the lander on loading payload is in a good condition, and to complete the subsequent missions smoothly.we can see the process of soft landing of Chang 'e 3 is mainly divided into six stages.

The first phase of the range of the main reduction section from the moon is $15 \mathrm{~km}$ to $3 \mathrm{~km}$. The major is slowing down in this phase. When the velocity is $v=v_{0}-a t$, The change of the height in this phase is

$$
h=\frac{1}{2} a t^{2},
$$

through the Hermite interpolation method,Which is the contact interpolation,based on the following character,note $h_{1}(t)$ as a node $t_{0}$.The Lagrange interpolation polynomial of $t_{1}$, let

$$
h_{2}(t)=h_{1}(t)+c\left(t-t_{0}\right)\left(t-t_{1}\right)=h_{0}-\frac{h_{1}-h_{0}}{t_{1}-t_{0}}\left(t-t_{0}\right)+c\left(t-t_{0}\right)\left(t-t_{1}\right) .
$$

So no matter what the value of coefficient $c$.It always has 


$$
h_{2}\left(t_{0}\right)=h_{0}, h_{2}\left(t_{1}\right)=h_{1} .
$$

Again use condition $h_{2}^{\prime}\left(t_{0}\right)=h_{0}^{\prime}$ to determine the coefficient $c$.The result is

$$
h_{2}(t)=h_{0}+\frac{h_{1}-h_{0}}{t_{1}-t_{0}}\left(t-t_{0}\right)+\frac{1}{t_{1}-t_{0}}\left(\frac{h_{1}-h_{0}}{t_{1}-t_{0}}-h_{0}^{\prime}\right)\left(t-t_{0}\right)\left(t-t_{1}\right) .
$$

Through the above formula calculation, we can see the first stage of the trajectory equation is

$$
h_{2}(t)=3.42 t^{2}-1690.18 t+15000, h_{2}(t)=3.42 t^{2}-1690.18 t+15000 \text {. }
$$

The second stage of coarse phase of coarse obstacle avoidance obstacle avoidance range from the moon is $2.4 \mathrm{~km}$ to $100 \mathrm{~m}$ range,Its main is required to avoid large craters, carrying out landing site hovering above $100 \mathrm{~m}$ in the design of implementation, and Primary definition.The fall in place,It has the thick with reverse thrust acceleration and acceleration of gravity in the process of obstacle avoidance, The rough height change during the process of obstacle avoidance function is $h(t)=0.587 t^{2}$,To consider Newton interpolation formula is

$$
h_{2}(t)=f\left(t_{0}\right)+\frac{f\left(t_{1}\right)-f\left(t_{0}\right)}{t_{1}-t_{0}}\left(t-t_{0}\right)+c\left(t-t_{0}\right)\left(t-t_{1}\right) .
$$

on account of $h_{0}(t)=f\left(t_{0}\right)$ can be seen as Zero interpolation polynomial, On that for

$$
h_{2}(t)=h\left(t_{0}\right)+\frac{h\left(t_{1}\right)-h\left(t_{0}\right)}{t_{1}-t_{0}}\left(t-t_{0}\right)+c\left(t-t_{0}\right)\left(t-t_{1}\right) .
$$

Through the linear interpolation formula, we can calculate the track for the second stage,it is

$$
h(t)=-0.41 t^{2}+442.35 t-103881.36 .
$$

The third stage is hovering stage.The hovering stage for detector for safe landing is at the altitude of 100 meters,On the ground, so as to select safe landing site, the time changes and the path does not change in the process, that is

$$
h(t)=0 \text {. }
$$

The fourth stage is the Pure phase of obstacle avoidance.To start the attitude thruster in the process, Be careful to avoid the surface barrier, The process using 22 seconds downward 70 meters displacement.It has the gravity from the moon to chang 'e 3 and it also has the recoil force from chang 3 onto the ground in the process. By Newton interpolation it has

$$
h(t)=-25 t^{2}+32226.75 t-10379412.96 \text {. }
$$

The fifth stage is slowing down and freeing fall.The interval of slowing decline phase from the moon is $30 \mathrm{~m}$ to $4 \mathrm{~m}$.The main task of this phase is to control the lander with the distance from the moon $4 \mathrm{~m}$ and its speed is $0 \mathrm{~m} / \mathrm{s}$.A slowing process, the engine thrust upward, The acceleration produced in engine is contrast to the acceleration of gravity and is less than the acceleration of gravity,Reasoning calculated by engine produced in the opposite direction of the acceleration, Can be calculated by

$$
h(t)=-5 t^{2}+6881.32-223970,
$$

In the freeing fall stage, chang'e 3 is only affected by the moon's gravity,The acceleration of gravity of the moon is $g=1.6 \mathrm{~m} / \mathrm{s}^{2}$, So use the newton interpolation on the formula can has 


$$
h(t)=-69.2 t^{2}+98125.42 t-34774356 .
$$

Based on the above five stages of comprehensive we can get the landing orbit in different time is

$$
h(t)=\left\{\begin{array}{ll}
3.42 t^{2}-1690.18 t+15000 & 0<t<507 \\
-0.41 t^{2}+442.35 t-103881.36 & 507<t<632 \\
0 & 632<t<657 \\
-25 t^{2}+32226.75 t-10379412.96 & 657<t<679 \\
=-5 t^{2}+6881.32-223970 & 679<t<698 \\
-69.2 t^{2}+98125.42 t-34774356 & 698<t<720
\end{array} .\right.
$$

The optimal control strategyat at each stage. The first stage is to coarse adjustment the process of obstacle avoidance of the lander for chang 2,According to the crude produced in the process of obstacle avoidance for task requirements.Flight to security in the process of imaging sensors can be sustained observation scheduled landing area, the lander can pass the final free fall which would reach its scheduled landing.Considering the $7500 \mathrm{n}$ is not a visible area of the main engine plume that bring to half cone Angle is about 25 degrees of the cone, and the imaging sensor field is 30 degrees, in order to avoid the main engine plume that influence on imaging sensors, and make the line of the sight of imaging sensors with the distance as short as possible, take the imaging sensors view bias Angle of 40 degrees.In order to ensure that in the field of imaging sensors that can be observed the landing zone, the determination by falling trajectory approaching and horizontal Angle of 45 degrees straight line descend gradually close to the landing zone.

The second stage which according to the first phase of making the imaging sensor is adjusted to the surface of the moon,the lander according to imaging sensors determine whether there is a great influence to give way, choosing a relatively smooth terrain can make the lander continues to fall.

The main task of the third stage is carring out on the surface and high precision three-dimensional imaging, accurate detection of the landing area obstacles, determine a safe landing.According to the requirement of laser $3 \mathrm{~d}$ imaging sensors work scope restrictions and observation is big enough to the landing area,the selection of 90-110 m high hovering control range.To enter the hovering height of the initial navigation as hovering paragraphs navigation height control target as hovering navigation, offset by the variable thrust engine lander gravity, guarantee the stability of the lander at zero speed, posture hover state.Hover of controling objectives are: highly take into hovering period of navigation,the three directions target velocity is zero, the acceleration is zero;Imaging before horizontal speed control only, after imaging increased horizontal position controling. On the outside of the lander to the main engine and attitude maneuver controling, internal control utilization level of the engine.Three-dimensional imaging sensors in hover state, the visual field landing area of the three-dimensional imaging, the lander relative moon landing area for high resolution slant distance data information.

The fourth stage is to ensure the accuracy of the final placement of obstacle avoidance and save propellant, the accurate obstacle avoidance and falling of the lander are at the same time.According to determine a safe landing, down from about 100 meters high inclined 30 meters above the landing site, horizontal velocity is near zero.

The fifth stage is ensure the moon landing speed and precision of attitude control, slowly decline stage to use a smaller set speed uniform vertical drop, eliminate horizontal velocity and acceleration, keep the lander level position, until received shutdown sensors signals or acceleration measurement is greater than the preset value will shut down the engine, landed the lander in the form of free fall.Slow down the process of horizontal zero speed control, position control for the slow down when the lander position.Vertical direction, more than 20 meters high control the speed and acceleration;Height less than 20 meters, control acceleration and acceleration instruction only slightly less than the geographical position of the moon's gravity acceleration, improve the 
reliability of the lander safe drop.

\section{Conclusions}

Chang 'e no. 3 task is our key of the second phase of the moon.Chang 'e no. 3 task has three key tasks, one is on table topography and geology survey;Second, on material composition and available resources survey;Three is the earth plasmas layer on detection and optical astronomical observations.Month falls in dynamic process, the probe to undergo complex attitude control and orbit control, make dynamic precision molding.In this paper, based on the dynamics, using interpolation method, can accurate simulation soft landing process, to achieve a soft landing process real time orbit determination.

\section{Acknowledgements}

Research of Higher Education Science in Heilongjiang Higher Education Association(14Q140), Heihe University teaching reform project (Xjg1429, Xjg1437),The Heihe University youth research talents support plan

\section{References}

[1]Y.Q.Song, T. Zhang, Y. Lin and J. Liang. Chinese health education.Vol.3(2014),03:p.241-245. (In Chinese)

[2]Y.B.Fan:International Conference on Applied Sciences,Engineering and Technology (Qingdao,China,July 28-29,2014). Vol.8, p.1541.

[3]Y Li, X.H. Chen and P.F. Zhang: Chinese population·resources and environment, Vol. 17 (2007).3,p.50. (In Chinese)

[3]X.Z. Bao and X.F. Li: Scientific Research, Vol. 28 (2010) 2, p. 275. (In Chinese)

[4]Y Zhao: Southwest University for Nationalities (Natural Science Edition), 2008, 34 (5):132-133.

[5]H.Y. Chen. Academic frontier, Vol. 11(2013), p. 26.

[6]M Li. Russian demographic crisis. (MS., Hei Longjiang University, China 2010).

[7] Information on http://data.worldbank.org.cn/.

[8]P.Du, Z.W. Zhai and W. Chen: Population Studies, Vol.1 (2005) No.6, p.90-93. 